\title{
RESUMEN
}

El presente trabajo, tiene por objetivo primario definir el concepto de racionalidad y sus distintos tipos a través de la revisión de la literatura. Posteriormente, se plantea las racionalidades explicitas e implícitas en la formación de profesionales de la contabilidad, para finalmente describir un modelo que, en estudios posteriores, permitirá realizar un análisis de las racionalidades explicitas e implícitas presentes en la formación de profesionales contadores auditores en Chile.

Palabras Claves: Racionalidades, aprendizaje, prácticas pedagógicas.

\section{TIPOS DE RACIONALIDADES Y MODELO PARA SU IDENTIFICACIÓN EN PROCESOS DE ENSEÑANZA APRENDIZAJE DE CONTADORES AUDITORES}

Luis Felipe Madariaga $/$ Carlos Santos ${ }_{2}$ 


\section{TYPES OF RATIONALITIES AND MODEL FOR IDENTIFICATION IN TEACHING LEARNING PROCESSES}

\section{ABSTRACT}

The present work, has as primary objective to define the concept of rationality and its different types through the review of the literature. Subsequently, the explicit and implicit rationales are raised in the training of accounting professionals, to finally describe a model that, in subsequent studies, will allow an analysis of the explicit and implicit rationales present in the training of professional accountant auditors in Chile.

Keywords: Rationalities, learning, pedagogical practices. 


\section{INTRODUCCIÓN}

Históricamente el desarrollo de la teoría administrativa y la gestión empresarial, han tenido como foco el desarrollo de sistemas de gestión empresarial que permitan optimizar procesos y por ende maximizar los resultados obtenidos por las organizaciones, es decir generar empresas exitosas desde la óptica económica. En este sentido, la contabilidad se constituye en una de las herramientas que han contribuido al logro de estos propósitos, así el desarrollo de la ciencia contable y las técnicas aplicadas se han centrado en la generación de información para la toma de decisiones fundamentalmente de carácter económico, siendo recientes las preocupaciones por aspectos medioambientales y sociales.

Por otra parte, el desarrollo de la gestión empresarial y la contabilidad enfrentan actualmente lo señalado por Bauman (2003) "En un mundo de cambios fluidos, valores cambiantes y reglas eminentemente inestables, la reducción de los riesgos, combinada con la aversión a descartar otras opciones es lo único que queda de una elección racional". En este contexto, se requiere analizar el tipo de racionalidad que guía la formación de los profesionales de la contabilidad.

La creciente importancia de la información contable, no solo para efectos de la toma de decisiones empresariales, sino también para la sociedad, plantea la necesidad de conocer la o las racionalidades explicitas e implícitas presentes en la formación de los profesionales Contadores Auditores, y sus efectos sobre el aporte social de su quehacer profesional.

¿Los programas de formación de contadores auditores en Chile, consideran distintos tipos de racionalidades en sus procesos de formación?

¿Las racionalidades explicitas 0 implícitas en los procesos de formación de contadores auditores, se circunscriben fundamentalmente a racionalidades de tipo instrumental?

El presente trabajo, se enmarca en una primera aproximación a la identificación y análisis de las racionalidades explicitas e implícitas en la formación de profesionales contadores auditores, por lo que presenta en primer término una descripción del concepto de racionalidad, para posteriormente un modelo que posteriormente se puede utilizar para la identificación y análisis de las racionalidades presentes en los procesos de formación profesional.

\section{OBJETIVO GENERAL}

Describir el tipo los tipos de racionalidades y un modelo para identificar las racionalidades explicitas e implícitas contempladas en la formación contable. 


\section{OBJETIVOS ESPECÍFICOS}

Definir el concepto de racionalidad y su relación con los procesos de formación profesional.

Describir modelo de identificación de saberes y prácticas presentes en las prácticas pedagógicas con el propósito de aplicarlo a los procesos de enseñanza aprendizaje de la carrera de contador auditor.

\section{EL CONCEPTO DE RACIONALIDAD}

De acuerdo a lo planteado por (Kant, 1787) "La experiencia es, sin ninguna duda, el primer producto surgido el nuestro entendimiento", esto lleva a plantearse en el proceso mediante el cual se comprende determinando conocimiento, como se estudia y finalmente como se aplica. En este sentido, es Max Weber, quién desarrolla en profundidad el concepto de racionalidad y distingue la racionalidad de la razón de la ciencia, la cual plantea que se reduce su diversidad a la lógica analítico-calculista, además de formalizadora de todo contenido y riqueza de la totalidad, lo que implicó posteriormente que racionalidad comienza a quedar fuera de su jurisdicción y, de este modo, catalogado de no-racional o irracional (Salvat, 2014).

Para Habermas, la racionalidad no es más que un caso particular de una idea más amplia de razón que tiene su modelo en la comunicación lingüística. Su manera de entender la racionalidad tiene menos que ver con el conocimiento 0 con la adquisición de conocimiento que con la forma en que los sujetos, capaces de lenguaje y de acción, hacen "uso" del conocimiento. (Habermas, 1989: 24).

Posteriormente Habermas, plantearía la racionalidad como una propuesta a partir de la argumentación para arribar a una teoría de la acción comunicativa (Habermas J. , 1989). Es por lo señalado, que, en primer en primer término, es necesario remitirse a la etimología, la palabra racionalidad, tiene su génesis en el latín "ratio" (razón o proporción), este a su vez, es la traducción del término griego "logos" (razón), que deriva del verbo "legein" (recoger, ordenar, pensar, leer), lo que en definitiva se constituye en una forma de vincular pensamientos o conceptos. En este sentido Habermas (1987) plantea que la racionalidad esta menos relacionada con el conocimiento o con la adquisición de este, sino que se relaciona directamente con las formas en que las personas a partir del lenguaje y la acción hacen uso del conocimiento. En la misma línea (Tua Pereda, 1988) señala que el concepto "racionalidad" deriva en la capacidad de dar razones, de poder respaldar las palabras y calcular las acciones, es decir la capacidad de cálculo y argumentación.

Por lo anterior, es importante comprender la distinción entre razón y racionalidad, la primera se puede definir como la facultad que tienen los hombres para poder 
asimilar intelectualmente el mundo y su naturaleza (Giraldo Garcés G. , 2007). Lo anterior permite diferenciar el concepto de razón del de racionalidad. Esta última corresponde a una categoría que permite identificar cómo los hombres reconocen y llevan a cabo sus fines. Así también se puede definir, en su acepción amplia, como la propiedad de los procesos y fenómenos de llevar a un determinado resultado. Es por lo anterior, que la literatura clasifica y define la racionalidad como una categoría filosófica que permite la identificación precisa de los medios y los fines. Lo anterior, permite establecer el hecho que la potestad de utilizar la razón la poseen todos los seres humanos. Por otra parte, no todos los seres humanos y sus actividades, trabajo y labores tienen el mismo grado de racionalidad. Es así, como las racionalidades pueden entenderse también como una categoría filosófica que describe el mecanismo por el cual opera el proceso de la identificación y la elección de los medios más adecuados para alcanzar un fin u objetivo específico. Es así, que, bajo esta definición, los fines se entienden como dados, es decir, no necesitan ninguna justificación y no son cuestionados. En el mismo sentido (López \& Salazar, 2013) plantean que las racionalidades permiten dar cuenta de un orden de significación de nuestros actos, es definitiva corresponde a la explicación de nuestras actuaciones. Por lo anterior, se afirma que la racionalidad media las relaciones sociales al entenderse como un conjunto específico de supuestos y prácticas en los que subyace siempre unos intereses que definen y valoran el reflejo de las personas en el punto, por lo que se encuentran condicionados y condicionan desde las expectativas generadas al respecto como referente epistemológico (Giroux, 1998).

De acuerdo a Kalberg (2005), se pueden distinguir cuatro tipos de racionalidad:

a) La racionalidad práctica: es aquella que persigue intereses puramente pragmáticos, individuales y egoístas aceptando las condiciones que emergen de la realidad que se considera como dada vinculándose con la capacidad del hombre de actuar racionalmente de acuerdo a medios-fines.

b) La racionalidad teórica: refiere a la capacidad de producción de conceptos abstractos precisos que permitan una comprensión consciente - cognitiva — de la realidad, este tipo de racionalismo no se da en el ámbito de la acción sino más bien en el del pensamiento sin que esto signifique que la confrontación teórica con la realidad no pueda introducir nuevas o diferentes regularidades de acción rutinaria.

c) La racionalidad sustantiva: se sustenta sobre un postulado de valor que hace de "medida de valor" o patrón de acción sobre el "torrente interminable de eventos empíricos de la realidad", vinculándose directamente con las acciones racionales con arreglo a valores.

d) La racionalidad formal: hace referencia estrictamente a esferas de vida y estructuras de dominación típicas de las sociedades occidentales modernas, industrializadas, sobre la base de la economía racional (cálculo y previsibilidad) 
y la administración —dominación - burocrática, es un tipo de racionalidad en apariencia similar al práctico ya que comparten su estrecha vinculación con las acciones con arreglo a medios-fines, pero orientadas de forma universal (en el sentido de "sin consideración de las personas") sobre la base de estatutos, reglas formales y mecanismos de cálculo racionales y burocráticos.

A partir de las distinciones de anteriores, se puede identificar o tipificar las racionalidades presentes en los distintos contextos históricos, sociales y en los procesos formativos. Es así, que Trovero (2012) señala que actualmente podemos vislumbrar el tipo de racionalidad formal, asociado al particular momento histórico (digamos la "modernidad-occidental" o la "época capitalista") en el cual el proceso de racionalización creciente abarca todas las dimensiones de la vida y sus estructuras.

Según Nicholas Rescher (1995), la racionalidad debe estar encaminada a disminuir la incertidumbre, ya que ella permite evaluar y ponderar las creencias que se tienen sobre un hecho cualquiera, que hasta el momento se daban por establecidas o con un sentido de irrefutabilidad, dependiendo de las circunstancias y las posibilidades vigentes. Es por lo anterior, que la racionalidad tiene entre sus funciones otorgar a los profesionales un instrumento que les permita evaluar ciertos conceptos, teorías o hechos presentes. Entonces, el papel fundamental de ésta, es permitir la "evaluación", la "reflexión" sobre los comportamientos individuales y grupales de una sociedad, al igual que sobre las elecciones de razones y argumentos para construir conocimiento, tomar decisiones y guiar acciones.

El hecho que las racionalidades se adecuen al contexto histórico y las realidades particulares, además de relativizarse de acuerdo a los propósitos de los diferentes contextos, autores como Weber y Rescher han propuesto nuevas clasificaciones de racionalidades:

a) La racionalidad cognoscitiva: Permite determinar lo que se ha de aceptar en un determinado momento, pues exige el uso de razones poderosas, teorías e informaciones, para la aceptación de creencias; su fin es el conocimiento puro. Según Rescher (1995), esta racionalidad se convierte en el mejor camino disponible para asegurar respuestas racionalmente coherentes.

b) La racionalidad práctica: Su esencia es la eficacia de los medios y la validez de los fines. La racionalidad práctica se orienta al ejercicio de acciones racionalmente adecuadas. Lo adecuado aquí es lo realmente accesible dada la información incompleta al alcance. Debe en consecuencia fundarse en el apoyo cognoscitivo apropiado de la información debidamente explorada.

Por otra parte, Weber (1964) propone una clasificación con carácter sociológico al realizar un cuestionamiento sobre el sentido de la acción social de los hombres.

a) Racional con arreglo a fines: Está determinada por expectativas en el comportamiento tanto de objetos del mundo exterior como de otros hombres, y 
utilizando esas expectativas como condiciones o medios para el logro de fines propios racionalmente sopesados y perseguidos.

b) Racional con arreglo a valores: Determinada por la creencia consciente en el valor- ético, estético, religioso o de cualquier otra forma como se le interpretepropio y absoluto de una determinada conducta, sin relación alguna con el resultado, o sea puramente en méritos de ese valor.

c) Afectiva: aquélla especialmente emotiva, determinada por afectos y estados sentimentales actuales.

d) Tradicional: Determinada por una costumbre arraigada.

Se debe tener en consideración, que esta tipología se refiere a tipo ideales y que, en la realidad -según lo explicita el mismo (Weber, Economía y Sociedad, 1964) "muy raras veces la acción, especialmente la social, está exclusivamente orientada por uno u otro de estos tipos".

\section{RACIONALIDAD INSTRUMENTAL 0 CON ARREGLO A FINES Y CONTABILIDAD}

El administrador de empresas necesita conocer las realidades que influyen en las organizaciones porque estas son su campo de intervención profesional. Para conocer necesita investigar, y según su conocimiento, interviene mediante la toma de decisiones. (López \& Salazar, 2013). En términos de la o las racionalidades que definen las acciones en términos de los procesos administrativos, se podría señalar que corresponden a racionalidades de tipo instrumental, debido a que se encuentran guiadas por el resultado que tendrá la acción que se realiza (Elster, 1996).

De acuerdo a Rescher (1993), la racionalidad instrumental tiene relación con la información real, es decir, con el tipo de movimientos y medidas que conducen a los fines de manera. Por lo anterior, no es posible señalar que la racionalidad instrumental no es lo contrario de lo que se ha conceptualizado como razón, de cierto modo es su consecuencia, ya que la racionalidad en la modernidad se inclinó hacia una tendencia que priorizó la utilización de instrumentos fáciles que correspondían a una explicación de la realidad. Las racionalidades de este tipo poseen implícita una visión económica, lo que produce que los medios sean calculados y definidos a partir de la relación costo-beneficio que nos proporcionan. Algunos economistas se inclinan en considerar que lo central de la racionalidad es la maximización de la utilidad; esta utilidad se caracteriza por ser mensurable y tiene una relación directa con la satisfacción y el bienestar. La medición y la comparación resultan esenciales para la maximización de la utilidad.

Al basarse en las diferentes categorías de racionalidad, se podría inferir que la contabilidad se enmarca en "racionalidad formal con arreglo a fines". Así también 
lo señala León (2008), quien señala que la contabilidad corresponde a una racionalidad instrumental en el que los fines vienen dados a través de datos para ser procesados a través de un algoritmo matemático (la partida doble). Lo anterior, también se fundamenta a partir de los propósitos de la contabilidad (la información y el control), fue en ellos qué la contabilidad recurrió al desarrollo de la "partida doble", la que como señala León (2008) se adscribe como herramienta matemática de aprehensión de la realidad.

\section{FORMACIÓN EN CONTABILIDAD}

Los programas de contabilidad y auditoría ofrecen varios módulos de contabilidad, incluyendo contabilidad financiera, análisis de estados financieros, contabilidad de gestión y control de gestión. Además de asignaturas de formación general e inglés, las que van confluyendo con el propósito de lograr el perfil de egreso definido por la institución de educación superior.

Para (Rorty, Educación sin Dogma, 1990) La educación superior no es cuestión de inculcar 0 sacar a luz la verdad. Más bien se trata de incitar la duda o estimular la imaginación, poniendo así en tela de juicio la opinión predominante. En este sentido, en general la contabilidad se aleja de este paradigma, por ejemplo de acuerdo (Carruthers \& Wendy , 1991) La contabilidad no es una actividad glamorosa; es repetitiva, orientado al detalle y metódica, no es un tema que acelere el pulso. Parece que la contabilidad es tan emocionante como sumar una larga columna de números. Los postulados de Weber son los más conocidos en términos de las racionalidades presentes en la contabilidad, y la define como la contabilidad del capital y la distingue como un componente crucial de su definición del capitalismo moderno. De acuerdo a Weber "El presupuesto más general para la existencia de este capitalismo actual es el de la contabilidad racional del capital como la norma para todas las grandes empresas industriales que se ocupan de satisfacer las necesidades cotidianas", además plantea que la "contabilidad racional del capital" implica la valoración y verificación de las oportunidades de lucro y del éxito de la actividad lucrativa mediante una valoración de los activos totales (bienes y dinero). En este sentido, La contabilidad hace posible que los capitalistas evalúen racionalmente consecuencias de sus decisiones pasadas. Pueden calcular exactamente los recursos disponibles actualmente para ellos y los que vendrán en el futuro. Lo que también implica que los propietarios del capital puedan usar la información proporcionada por una empresa para evaluar y comparar varias alternativas para las inversiones. (Weber, Historia Economica General, 1927)

En la misma orientación (Acevedo, 2017) plantea que la reducción de las personas a material humano y la paulatina marginación de los modos de pensar que no se inscriben dentro del rígido marco del pensamiento calculante 0 técnico, ha implicado que otros tipos de racionalidades vayan situándose en la periferia. Un ejemplo de lo anterior, es el actual sistema de educación superior en contabilidad, 
el que se basa en un aprendizaje activo por parte del estudiante enfocado al desarrollo de competencias genéricas y específicas. (Calabor, Mora, \& Moya, 2018). En el mismo sentido Giraldo (2007) señala "Un patrón de pensamiento que se ha generalizado es que todas las decisiones, personales y empresariales, impera la lógica del lucro. La contabilidad no ha sido ajena a dicha influencia ya que ésta se ve inmersa en una racionalidad de tipo instrumental". Es en este sentido, surge la pregunta respecto a las racionalidades explicitas e implícitas en la formación de profesionales del área de la contabilidad y auditoría. Por lo anterior, se puede establecer que el supuesto básico que circunscriben el conjunto de normas contables son útiles en la medida que logren alcanzar los objetivos informativos declarados para la contabilidad empresarial (renta, riqueza, variación de la riqueza, etc.) es la racionalidad económica de las diferentes transacciones empresariales, mientras los rasgos sociales y profesionales actuarán en todo caso como restricciones o matizaciones de segundo orden para la configuración de las soluciones contables idóneas.

En este sentido, el papel que desempeña la contabilidad en la toma de decisiones racional es también enfatizado en los libros de texto de contabilidad moderna. Las cuentas proporcionan información técnica sobre el resultado de acciones comerciales previas (Littleton \& Zimmerman, 1962). A través de dichos procedimientos, los inversionistas pueden evaluar de forma precisa el éxito relativo de inversiones particulares y, por lo tanto, facilitar la búsqueda de mayores ganancias (Istvan \& Avery, 1979). La opinión opuesta, es que la práctica contable no proporciona una escala objetiva de valuación que pueda ser utilizada para la toma de decisiones óptimas y para la evaluación de la gestión administrativa. Así también, se señala que la teoría contable tiene un carácter dogmático más que hipotético-científico y que sirve satisfactoriamente sólo a propósitos de naturaleza jurídica. Aún no ha logrado integrar los aspectos micro con los aspectos macro de las mediciones de ingreso-riqueza (Mattessich, 2002).

Al volver a revisar lo señalado por Weber (1964) y relacionarlo con la contabilidad como una disciplina de la economía, el plantea que la premisa de la racionalidad del capitalismo se fundamenta, en el irracional afán de lucro ilimitado, 0, por lo menos, con su contención racional. Al plantear el proceso de la racionalidad, lo entiende como un proceso de matemático progresivo de toda la experiencia y todo conocimiento que, a partir de sus espectaculares éxitos en las ciencias naturales, se orientan a la conquista de las ciencias sociales y, por último, a la del propio modo de vida, es decir, a una cuantificación universal hecho que se puede observar claramente en la contabilidad y en general de las disciplinas que configuran el saber contable. Así también León (2014) plantea que la contabilidad, tiene una importancia sustantiva en la construcción de la génesis y el desarrollo de la racionalidad del capital. Mientras que Husillos (Husillos, 2004) profundiza esto al señalar que la racionalidad contable es una racionalidad formal, con arreglo a fines.

En el caso de Chile, la formación de Contadores Auditores en su rol profesional y por otro lado su rol social, debería tender a evitar pérdidas sociales producto de la 
existencia de asimetrías de información, es uno de los objetivos de la profesión de contador auditor. Así también, la generación de información relevante para la toma de decisiones. En este sentido, el desarrollo del mercado de capitales chilenos y la globalización de las empresas implicó un creciente desarrollo y demanda de profesionales de la especialidad, así también incremento los desafíos de formación de estos profesionales.

Hoy, la profesión enfrenta una serie de desafíos de formación, los que no sólo son impulsados por los requerimientos del mercado, sino también por factores sociales, medioambientales y gobernanza (sostenibilidad). Lo anterior, implica que el proceso de enseñanza aprendizaje deba hacerse cargo de estos desafíos e incorporar nuevos saberes y prácticas, mejorar los existentes o profundizar en algunos de ellos. Precisamente la auto-afirmación debe abrir los horizontes de sentido para moverse como pez en el agua en esas redes y brindar elementos de juicio para buscar permanentemente nuevos caminos a través del desarrollo de una comunicación capaz de concertar y manejar disensos. Aquí es donde se encuentra presencia de racionalidad, es allí donde se descubre esencia, crecimiento y desarrollo humano. Es en el espacio abierto de la racionalidad y por tanto de la auto-formación, donde la naturaleza humana, en medio de su contingencia, puede descubrir caminos para la identidad en el lenguaje y en el encuentro con otros. (Roa Várelo, 2005)

\section{RACIONALIDADES PRESENTES EN LAS PRÁCTICAS PEDAGÓGICAS.}

Ser racional es ser metódico: es decir, tener criterios de éxito fijados de antemano (Rorty, Objetividad, relativismo y verdad, 1991). En ese contexto, se pueden distinguir tres tipos de procesos que constituyen, típicamente, la forma de razonar bajo supuestos de racionalidad limitada. En otras palabras, de lo que podemos hablar es de tres conjuntos de heurísticos o reglas individuales de decisión que guían los procesos de toma de decisiones. Se trata de reglas simples de búsqueda, reglas simples de parada y reglas simples de decisión. Mediante las reglas de búsqueda, los individuos van incorporando unidades de información una a una, integrándolas junto a la información ya almacenada. (Gigerenzer \& Reinhard, 2001)

La presente investigación, se constituye en la primera etapa de análisis de las racionalidades presentes en la formación de profesionales de la contabilidad. Con este propósito, se utilizará le modelo planteado Latorre (2004), además de ir incorporando algunos aspectos relativos al contexto socio histórico de las últimas décadas. El modelo señalado, se articula a partir de cuatro componentes principales:

Para el análisis se requiere primero comprender el concepto de racionalidad definido con anterioridad, el cual además se puede reflejar en lo señalado por Latorre (2004) que lo asocia a estar dotado de sentido y no derivado del azar. Consecuentemente, los economistas clásicos, postulaban que las decisiones de 
los agentes económicos se fundamentan en la realización de cálculos efectuados a partir de los conocimientos y saberes de que se dispone hasta ese momento y lo que se sabe sobre la situación en la cual se debe actuar. Estos dos elementos, permiten a las personas determinar la solución más óptima y que es la mejor posible de encontrar, siendo la que le proporciona menor costo y mayor beneficio. Por lo anterior, se le denomina "racionalidad perfecta", "objetiva", "instrumental" 0 "utilitarista". En este sentido, es posible también remitirse a la teoría de Teoría de la Elección Racional (TER), conocida también como "modelo de la utilidad esperada" que fue planteada por Gary Becker (1996) y anteriormente por Coleman (1986), quienes además plantean que dicha teoría es válida para todas las ciencias sociales. Al cumplirse esta teoría implica que las personas realizan acciones porque piensan que van a proporcionarles una satisfacción máxima.

En el contexto de la práctica pedagógica, específicamente en lo que tiene relación con el currículo, se pueden distinguir tres intereses cognitivos básicos (Habermas J., 1992): el interés técnico, el práctico y el emancipador 0 crítico. El interés técnico, se justifica en el dominio instrumental de la naturaleza y da lugar a las ciencias empírico-analíticas; el interés práctico, parte de la comprensión intersubjetiva entre los seres humanos, fundamentan las ciencias históricas-hermeneúticas centradas en criterios interpretativos y el interés emancipador, fundamentan las ciencias sociales y filosóficas, centradas en criterios de verdad emancipadores. En materias contables se ha señalado En lo pedagógico, la enseñanza contable hace demasiado énfasis en los aspectos técnicos y no se preocupa por relacionar los nuevos logros científicos con el cuerpo establecido de conocimientos contables (Mattessich, 2002).

Por otra parte, Boudon (2003) señala que las buenas razones sobre las cuales se fundan las acciones individuales pueden ser de naturalezas muy diversas (emocionales, afectivas, por hábito o rutina, por adhesión a un valor, por adhesión a una norma), lo que hace posible tipificar estas racionalidades que las personas poseen y utilizan cuando se enfrentan a decisiones respecto a las acciones a desarrollar en diversas circunstancias.

Además de lo señalado con anterioridad, en la identificación de racionalidades, se debe considerar el hecho que estas presentan un carácter de situado, es decir son acordes a un contexto socio histórico, temporal y espacial determinado (Larorre, 2004). También es necesario considerar que las racionalidades no son solo abstracciones, sino estructuras de pensamiento que se encuentran incorporadas en contextos espaciales y temporales que es donde la persona ejerce las acciones. En este sentido, por ejemplo, es necesario analizar el entorno social y contexto donde se desempeñarán los futuros profesiones. En este aspecto, cobra sentida la incorporación del concepto de "modernidad liquida" (Bauman, 2003), de la cual señala que, de una sociedad sólida se pasó a una sociedad líquida, maleable, escurridiza, que fluye, en un capitalismo liviano. Lo anterior, en varios aspectos de sociedad, tales como: la ciencia, la tecnología, lo político, económico, intercambio cultural, apertura de mercados y la globalización. 
Otro elemento a considerar de acuerdo al modelo, es que las características del contexto situacional, así como la posición que la persona ocupe al interior de dicho contexto o sistema, influirá sobre el individuo, condicionándolo, aunque no determinando su pensar y actuar. En términos teóricos, Bajo este contexto, en este punto se incorpora también el análisis de las características de los procesos educativos, para lo cual se debe analizar las prácticas pedagógicas, ya que es ahí donde se encuentran los saberes, donde además se reúnen profesores y estudiantes y estructuran relaciones de diversa naturaleza: epistemológicas, didácticas y sociales.

\section{a) Naturaleza teórica - práctica de la acción educativa.}

De acuerdo al modelo, para el análisis de la naturaleza teórica-práctica de la acción educativa, se debe considera el concepto de acción individual, la cual tiene como objetivo que el hecho educativo se transforme en el centro de atención e interés principal de los actores del campo educativo; estas acciones serán privilegiadas para el estudio de las racionalidades implícitas en ellas.

El fenómeno educativo se encuentra dotado de una naturaleza dual, es decir, caracterizado por la coexistencia simultánea de dos componentes o dimensiones en su interior: una teórica y otra práctica. Estos componentes o dimensiones se expresan a través de la existencia de dos constructos al interior de todo hecho educativo: una teoría de la educación y una práctica educativa. En otras palabras, cada vez que se observa, estudia 0 analiza un hecho educativo es posible reconocer que en él están presentes teoría y práctica a la vez (Ferry, 1990).

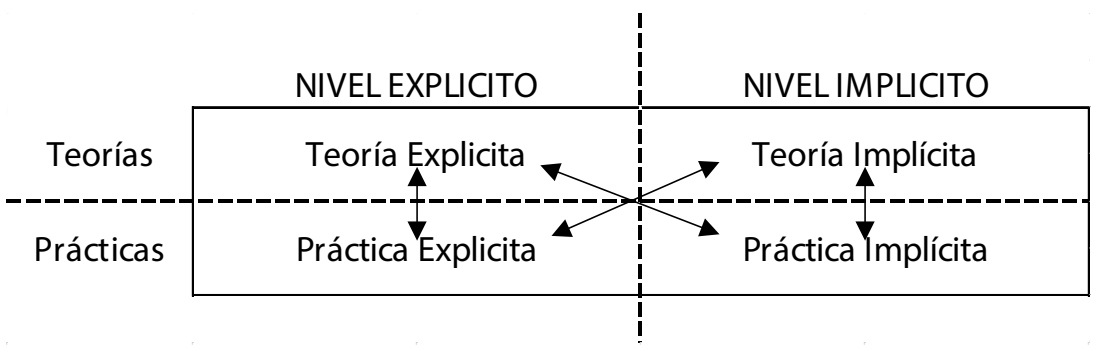

Fuente: Latorre (2004)

Teoría y práctica coexisten y se relacionan, pero no de una manera simple. El tipo de relación que se establece entre teoría y práctica no posee las características de un vínculo directo, susceptible de ser definido por características de dominaciónsubordinación de una sobre otra. Por el contrario, las características de las relaciones entre teoría y práctica educativas son complejas, pues refieren a vínculos de mutua superposición (Latorre, 2004). Además, esta mutua superposición entre teoría y práctica se produce en dos niveles: uno implícito y otro explícito. Es decir, en el contexto del proceso educativo se encuentran teorías explícitas y teorías implícitas, además de coexistir, prácticas explícitas y prácticas implícitas. Por lo anterior, es que las relaciones antes señaladas deben ser analizadas para poder identificar las racionalidades explicitas e implícitas en los procesos de formación. 


\section{b) Teorías y saberes asociados a la acción educativa.}

En este punto, el modelo se centra la noción de teoría, la cual se remite a una estructura organizada de conocimientos, por otra parte, la noción de saber da cuenta de un cuerpo heterogéneo de conocimientos que difieren tanto en su naturaleza epistemológica como en sus modos de adquisición y niveles de formalización. A partir de esta distinción, se plantea que los actores educativos poseen un cuerpo de saberes diversos a partir de los cuales elaboran sus teorías.

\section{c) Características de las prácticas pedagógicas.}

En el contexto de la práctica pedagógica, específicamente en lo que tiene relación con el currículo, se pueden distinguir tres intereses cognitivos básicos (Habermas J. , 1992): Interés técnico, práctico y el emancipador o crítico. De este modo, el tipo de conocimiento generado por el interés técnico requiere de ser objetivo, verificable por observación empírica, replicable, generalizable y predictivo. En este tipo de saber se da una relación de subordinación vertical entre la teoría y la práctica. predictibilidad del saber ocupa un lugar central en este tipo de conocimiento, en cuanto a través de ella se puede "anticipar cómo será (probablemente) el ambiente futuro a partir del conocimiento en su forma actual. Así mismo, en potencia, permite controlar dicho ambiente sobre la base de ese saber". Existe pues una relación directa entre conocimiento, control y poder y, según Habermas, "el significado último del conocimiento predictivo consiste en la posibilidad de la explotación técnica de dicho conocimiento".

De acuerdo a Latorre (2004), las nociones de acción y práctica educativas son utilizadas de manera similar y para referirse a una misma realidad. La acción ha sido definida de maneras diversas; para algunos corresponde simplemente a la condición opuesta a la condición de pasividad, en otras palabras, quien actúa deja de ser pasivo. La noción de práctica, entonces, aparece como un adjetivo que califica un tipo particular de acción. Para algunos autores, desde una perspectiva conductista, práctica es la acción humana que se realiza en un contexto material concreto, práctico; para otros, en cambio, desde una perspectiva más sociológica y antropológica, la definición de práctica corresponde a la de acción humana dotada de sentido, objetivos y significados para quien la realiza. En el contexto de esta investigación, la práctica educativa y, más específicamente, las prácticas pedagógicas serán consideradas desde una doble perspectiva, tanto desde la acción realizada como desde el sentido atribuido a dicha acción por quien la realiza. Esta doble entrada hará posible articular un nivel descriptivo con otro comprensivo.

La práctica pedagógica es explicada como la suma de efectos de acciones individuales; esto abre el espectro de su consideración como resultado deseado e intencionado o resultado no esperado, no deseado o no percibido de manera consciente por los actores individuales. En este sentido, el objetivar las racionalidades presentes en las prácticas pedagógicas hará posible dar cuenta del mecanismo estructurador u organizador del conjunto de acciones que realiza un 
individuo y que se expresa concretamente a través de las acciones que este realiza (Pascual, 1998).

Es así, que la enseñanza de la contabilidad comprendida como ciencia debería tender a como cualquiera disciplina científica, incluso aquellas que se especializan en problemas de conocimientos tecnológicos y técnicos, realizar una comprensión del medio en el cual se desata la transmisión de saberes, de tal forma que pueda desarrollarse favorablemente el proceso. Lo anterior implica un estudio juicioso y comprometido de quienes aprender, enseñan y educan de forma coherente con las necesidades de la sociedad y no únicamente con aquellas derivadas del mercado de capitales (Gómez, 2015).

Por lo anterior, es que es necesario una revisión de las prácticas pedagógicas y las racionalidades formadas en los procesos de enseñanza aprendizaje, los cuales permitirán a los estudiantes tomar decisiones en su ámbito laboral.

\section{CONCLUSIONES}

Este trabajo corresponde a una etapa preliminar de identificación de las racionalidades presentes en la formación de profesionales de la contabilidad y auditoría. En este contexto, esta primera etapa permite distinguir el concepto de racionalidad del concepto de razón, además de reconocer las definiciones de los distintos tipos de racionalidades descritas por la literatura, esto con el objetivo de posteriormente poder relacionar los distintos programas, contenidos y prácticas pedagógicas utilizadas en la formación profesional.

En este sentido, se identificó el modelo desarrollado por Latorre (2004), el cual describe los diferentes tipos de saberes y prácticas pedagógicas a través de las cuales se pueden identificar las racionalidades presentes en la formación de profesionales de la contabilidad. Lo anterior, se justifica desde la necesidad actual de contemplar en los procesos de formación no solo las racionalidades instrumentales (fines), sino también otro tipo de formas de análisis, acción y decisión, que permitan a los futuros profesionales hacer frente a los desafíos no solo del mercado laboral y de capitales, sino a los nuevos desafíos sociales, medio ambientales. 


\section{REFERENCIAS}

ACEVED0, J. (2017). Heidegger y la época técnica. Santiago: Editorial Universitaria.

BAUMAN, Z. (2003). Modernidad Líquida. México: Fondo de Cultura Económica.

BOUDON, R. (2003). Raison, bonnes raisons. París, Presses Universitaires. París: Presses Universitaires.

CALABOR, M. S., Mora, A., \& Moya, S. (2018). Adquisición de competencias a través de juegos seriosen el área contable: un análisis empírico. Revista de Contabilidad - Spanish Accounting Review, 38-47.

CARRUTHERS, B. G., \& Wendy , E. N. (1991). Accounting for Rationality: Double-Entry Bookkeeping and the Rhetoric of Economic Rationality. AJS, 31-69.

ELSTER, J. (1996). Rationality and the Emotions. Wiley on behalf of the Royal Economic Society, 1386-1397.

FERRY, G. (1990). El Trayecto de la Formación: Los Enseñantes entre la Teoría y la Práctica. México: Paidós.

GIGERENZER, G., \& Reinhard, S. (2001). Rethinking Rationality. En G. Gigerenzer, \& S. Reinhard, Bounded Rationality (págs. 1-12). London: The MIT Press.

GIRALD0 Garcés, G. (2007). La cosificación de la contabilidad. Contaduría Universidad de Antioquia, 133-154.

GIRALD0 Garcés, G. (2010). Contexto económico, ética y actitud crítica en la formación del contador público. Contaduría Universidad de Antioquia, 221-239.

GIROUX, H. (1998). Teoría y resistencia en educación. México: Siglo Xxi.

GÓMEZ, J. (2015). Prácticas pedagógicas: una mirada crítica. Visión Contable, 17-31.

HABERMAS, J. (1987). Teoría de la Acción Comunicativa. Madrid: Taurus.

HABERMAS, J. (1989). El Discuso Filosófico de la Modernidad. Madrid: Taurus. 
HABERMAS, J. (1992). Conocimiento e interés. Buenos Aires: Taurus.

HUSILLOS, F. J. (2004). Información medioambiental, contabilidad y teoría de la. "Nuevas Tendencias en Dirección de Empresas", 07/04.

ISTVAN, D. F., \& Avery, C. G. (1979). Accounting principles. (H. B. Jovanovich, Ed.) New York: Universidad de Michigan.

KALBERG, S. (2005). Utilizing Max Weber's 'Iron Cage' to Define the Past. The Protestant Ethic Turns 100, 191-208.

KANT , I. (1787). Crítica de la razón pura. Traducción de Pedro Ribas (13ª ed. ed.). Madrid: Alfaguara-Santillana.

LATORRE Navarro, M. (2004). Aportes Para El Análisis De Las Racionalidades Presentes En Las Prácticas Pedagógicas. Estudios Pedagógicos, 75-94.

LEÓN, G. (2008). De la "Contabilidad Sólida" a la "Contabilidad Líquida" ¿Crisis o Muerte de la Factualidad? Investigación Contable, 11-27.

LITTLETON, A. C., \& Zimmerman, V. K. (1962). Accounting theory: continuity and change. Englewood Cliffs: Prentice-Hall.

LÓPEZ, S., \& Salazar, G. (2013). La racionalidad en la toma de decisones. Grafías disciplinares, 41-55.

MATTESSICH, R. (2002). Contabilidad y métodos analíticos. Buenos Aires: La Ley.

MILLER, L. (2004). Acción colectiva y modelos de racionalidad. Estudios Fronterizos, 107-130.

PASCUAL, E. (1998). Racionalidades en la producción curricular y el proyecto curricular. Pensamiento educativo, 13-72.

RESCHER, N. (1995). Pluralism: Against the Demand for Consensus. OUP. Published to Oxford Scholarship Online.

ROA Várelo, A. (2005). Universidad, Mercado Laboral y Competencias: ¿Con qué nos quedamos? MEMORIAS DEL SEMINARIO INTERNACIONAL, (págs. 58-75). BARRANQUILLA.

RORTY, R. (1990). Educación sin Dogma. Facetas, 44-47.

RORTY, R. (1991). Objetividad, relativismo y verdad. (J. Vigil Rubio, Trad.) Barcelona, España: Ediciones Paidós Ibérica. 
SALVAT, P. (2014). Max Weber: Poder y Racionalidad. Santiago: RIL editores.

TUA Pereda, T. (1988). Evolución del concepto de contabilidad a través de sus definiciones. Contaduría Universidad de Antioquia, 9-74.

WEBER, M. (1927). Historia Economica General. (M. Sánchez Sarto, Trad.) México, México: Fondo De Cultura Económica.

WEBER, M. (1964). Economía y Sociedad. (J. Winckelmann, Ed.) México: Fondo de Cultura Económica.

WEBER, M. (2002). Economía y Sociedad. Madrid: Fondo de Cultura Económica. 